\title{
A Research of Wireless Environment Monitoring Equipment based on Lora Spread Spectrum
}

\author{
Luo Zhen, Shu Xinjian, An Zhiyuan \\ Information \& Telecommunication Co. of State Grid Henan Electric Power Company, Zhengzhou \\ 450052, China \\ E-mail:lowryhost@hotmail.com
}

Key words: Lora, Wireless, Environment Monitoring

\begin{abstract}
Cable is an essential component of Electric Power Secondary System. Due to the low voltage power cables lay with communication optical cable in the same trench, while low voltage power cables working current is high, communication optical cable suffers huge incidents potential of fire accident. This proposal intent to develop a Wireless Environment Monitoring Equipment based on Lora Spread Spectrum. This equipment includes three subsystems: water lever monitoring subsystem, temperature monitoring subsystem, and gas monitoring subsystem. The system realizes automatic alarm by real-time measurement of the temperature, water leakage and harmful gas content of the cable trench and vertical shaft. Therefore, ensures normal and reliable operation state of the cable and improves the reliability of electric grid supplements.
\end{abstract}

\section{Introduction}

Electric grid communication system is an important and indispensable component of power system, it is the foundation of the electric grid dispatching automation and modernization of production management and it is an important technical means to ensure the grid security, economic and stable operation.

As the state power infrastructure investment increased year by year, the length of the power tunnel, the length of the communication optical cable, and the number of vertical shaft also increased rapidly. However, the operation maintenance resource is far behind the growth rate of power-grid infrastructure, the power tunnel maintenance faces huge pressure. Cable trench is an essential infrastructure in substation and power plant, and cable in the trench works as the nerve center of power plant and substation. Once the optical cable fails, it will not only be difficult to check and handle the work, but also may result in the failure of relay protection and stability control, resulting in the expansion of the accident and the safety incident. Therefore, it is urgent to adopt modern technological means to improve operation maintenance level, timely and effectively determine potential hazards in the cable trench, vertical shaft or the telecom room to avoid the factors such as fire, water seepage which causes the damage of fiber optic cable and communication equipment. It is a great significance to maintain the security of electric communication network and power grid.

\section{System Structure Designation}

Due to the environment in cable trench and vertical shaft leads to wireless signal attenuation, most of transmission devices are realized by wired measures. But the cost is high, once the cable trench environment changes, the cable will no longer be reliable, so this device adopts the wireless communication mode. Lora is a dedicated technology to wireless spread spectrum modulation demodulation, it presents a striking contrast between other modulation scheme such as FSK (frequency shift keying), GMSK (Gaussian minimum frequency shift keying) and BPSK (binary phase shift keying) and its derived form. [1]

Previously, only a few of military communications systems were allowed to use these technologies. These technologies greatly improved the sensitivity of receiving this kind of means of communication, as well as the communication distance.[2] Therefore, this set of device which 
adopts Lora spread spectrum communication mode, perfect solved the problems of small amount of data from vast-distance communication in complex environment.

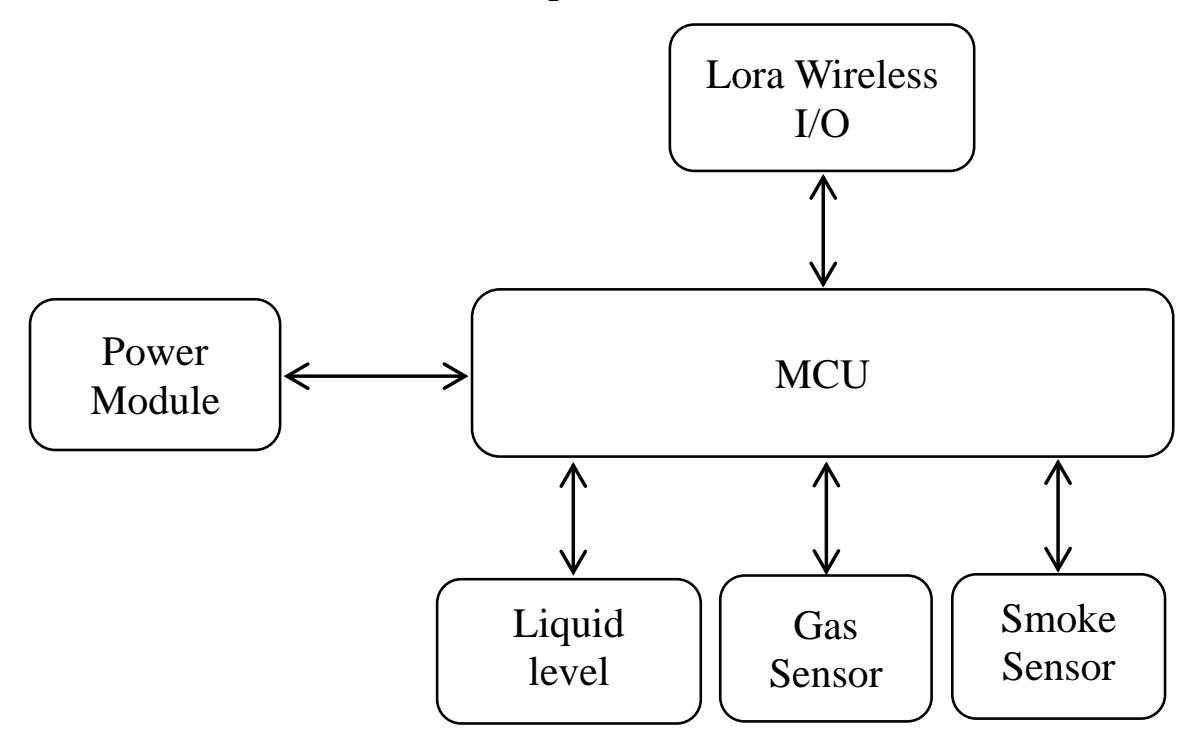

Fig.1 the functional structure of wireless Environment Monitoring Equipment

The environment monitoring device based on Lora spread-spectrum wireless long-distance employs modular design principle, which is mainly composed of power module, control module and sensor acquisition module. The power module is responsible for providing the entire equipment with the required working voltage and current. The control module is the brain of the whole device, which reads the data of each sensor and sends it to Lora gateway equipment placed in the computer room via Lora wireless technology.[3] The sensor collection module mainly includes liquid level sensor, gas sensor and smoke sensor. The system works by measuring the leakage of the cable trench and shaft and realizing automatic alarm. By analyzing the measurement results of smoke sensors, keeping the fire accidents away in the cable trench and shaft is achieved in real time. Through the detection of the content of combustible gas in the cable trench and shaft, the real-time understanding of the environmental state is realized to determine whether the underground hole can be safely constructed. The system adopts the modular structure, and each sensor can be combined as required. Each unit has a unique ID information around the world, at the time of installation using GPS devices labeled with binding in the background, in the process of running afterwards, according to APP embedded real-time map shows the location of the equipment information and running status, etc.

\section{Hardware Realizations}

Power Section. There are two types of power supply for the entire device: $5 \mathrm{~V}$ is responsible for powering external sensors, and $3.3 \mathrm{~V}$ is responsible for powering the core control chips and Lora communication chips. The conversion efficiency is up to 76 percent by adopting the imported industrial-grade switch power chip, with a voltage range of $6 \mathrm{~V} \sim 42 \mathrm{~V}$.

Sensor Acquisition Section. The liquid level sensor is the 4-20ma current signal output that converts from the liquid level height information, and the voltage signal is adjusted to $1-5 \mathrm{v}$ after the current-voltage conversion circuit on the device. The processor uses the internal AD conversion function to collect voltage signals, and the current level of liquid level is calculated according to the established by liquid-level-voltage mathematical model.

Smoke sensor converts the smoke concentration in air to $0 \sim 3.3 \mathrm{~V}$ voltage signal output, the processor uses the integration function of the $\mathrm{AD}$ conversion to calculate the voltage value. Thus, according to the established mathematical model of smoke concentration-voltage, the current the smoke concentration in air is deduced.

Combustible gas detection includes ammonia, methane and sulfide. The sensor is combined with I2C Bus interface and processer, to read the collection value through the I2C Bus protocol. Then it 
calculates the real combustible gas density, according to the sensor for the transition curve. [4]

Processing Core Section. Processing core section adopts STM32 processor, the chip not only integrates the necessary RAM and ROM program, but also integrates all kinds of common peripheral module and communication interface, including the AD conversion, I2C bus, and SPI interface for Lora communication as mentioned.[5] The official design references are provided to facilitate hardware design and software implementation. And each chip has a world-round unique-ID, which can be used to identify and encrypt data and secure data transmission.

Lora Communication Section. Lora communication uses SEMTECH Company's latest wireless chip of LoraTM modulation technology. This chip has the extreme distance spread spectrum communication, high anti-interference and the decrease of the maximum current power consumption, use LoraTM mode can realize - 148 DBM high sensitivity, and combined with the integration of + 20 DBM power output, can PK in the same industry of any kind of wireless chip, the chip is applicable to any complex environment of wireless data transmission applications. The chip USES the two-way SPI communication interface and the processor for data interaction.

\section{Software Realizations}

Considering the applying environments of the equipment, the equipment needs to fix with the power self-check function to determine the working conditions of the sensor, so as to facilitate early detection of possible problems. The flow chart of the program is as shown as Figure 2.

\section{Conclusion}

Through the designation and implementation of the hardware and software, as well as the final field test, the internal environment of uninterrupted monitoring function of the cable trench and shaft was achieved by Lora wireless technology. The cable is an important part of the secondary system of the power grid, and the guarantee of the normal and reliable operation of the cable has an inestimable effect on improving the reliability of power supply. Due to the low-voltage power cable groove concentrate with protection, control cable. While the low voltage power cables working current is big, in the event of fire accident, it is easy to spread with the communication optical cable, causing a total communication failure, so as to lead to an incident. This device is to ensure the safety of the shaft in cable and cable trench and reliable work of special measurement and control, which can effectively identify aging of cable and connector of overheating and fire accidents and other hidden trouble, using low cost of measure-control device in cable trench and the stability of the internal temperature of the shaft measurement, therefore, achieves a long-term recording and time data collection. Aiming to forestall and reduce risk.

\section{References}

[1] N. Sornin, M. Luis, T. Eirich. LoRa WAN Specification (V1.0.1 Draft 3). 2015

[2] Sornin N., Luis M., Eirich T., Kramp T., et al. LoRaWAN-(TM)Specification V1.0. 1st All Members Meeting and Open House. 2015

[3] Augustin A., Yi J., Clausen T., et al. A Study of LoRa: Long Range \& Low Power Networks for the Internet of Things. Sensors . 2016

[4] Yang Wang, Jiayu Li, He Jing, Qiang Zhang, Jingkun Jiang, Pratim Biswas. Laboratory Evaluation and Calibration of Three Low-Cost Particle Sensors for Particulate Matter Measurement[J] . Aerosol Science and Technology . 2015 (11)

[5] Meng Lieh, Sheu Wei-Hung, Hsu Lin-Jie Ts. A Capacitance-ratio-modulated Current Front-end Circuit With Pulse width Modulation Output for a Capacitive Sensor Interface. IEEE Transactions on Instrumentation and Measurement . 2012 


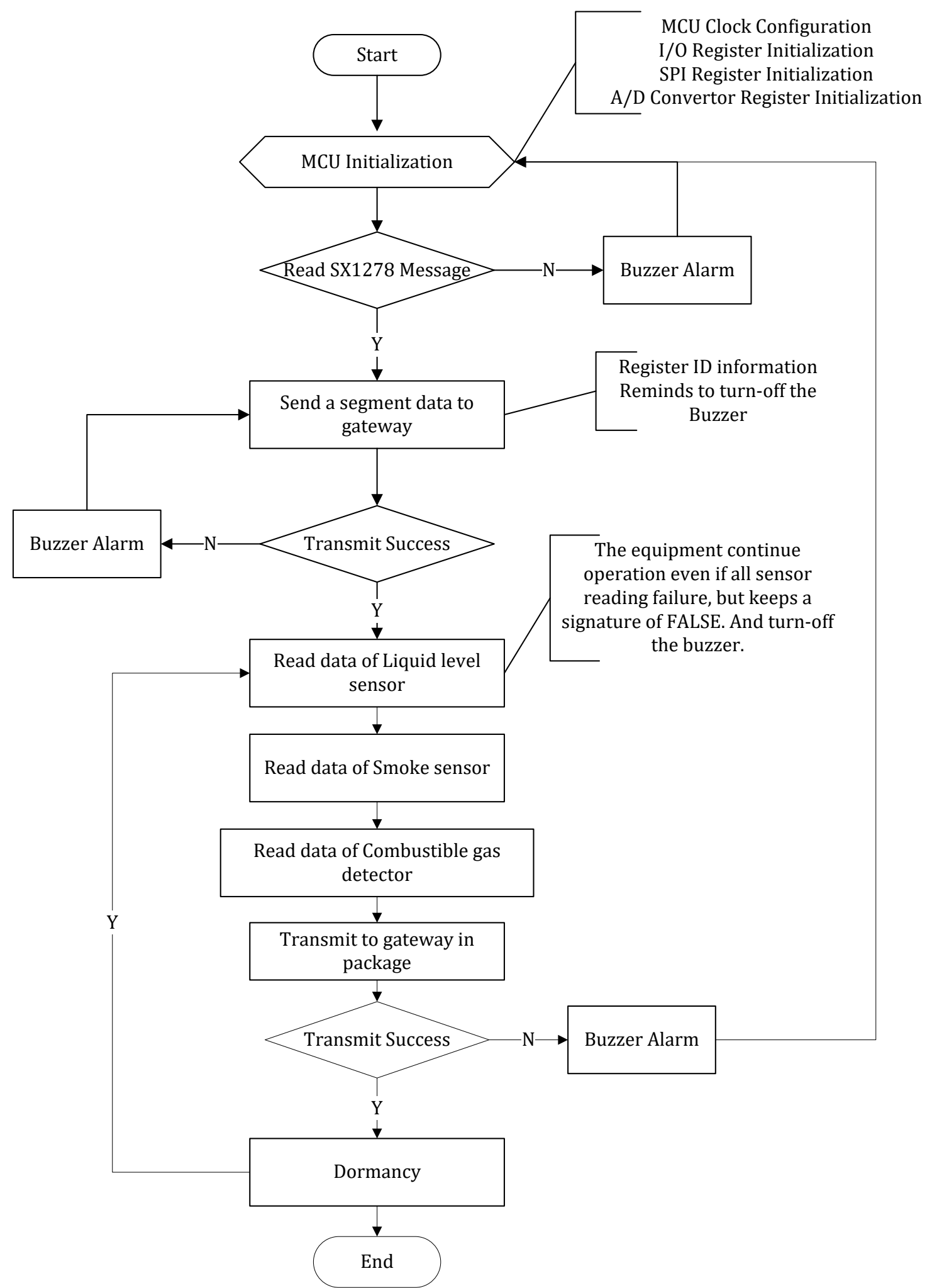

Fig.2 Software flow diagram 